\title{
Les contradictions de la gestion intégrée des ressources en eau dans l'agriculture irriguée méditerranéenne
}

Thierry Ruf ${ }^{1}$

Marie-Jeanne Valony ${ }^{2}$

${ }^{1}$ Institut de recherche

pour le développement (IRD),

Unité de recherche 168

Equipe Thermes

911, avenue Agropolis,

BP 64501,

34394 Montpellier cedex 5

<thierry.ruf@ird.fr>

${ }^{2}$ Centre national d'études agronomiques des régions chaudes (Cnearc),

1101, avenue Agropolis,

BP 5098

34033 Montpellier cedex1

$<$ valony@cnearc.fr>

\begin{abstract}
Résumé
Le modèle de gestion intégrée des ressources en eau repose sur des bases définies à Dublin en 1992 et reproduites sous des termes analogues depuis 15 ans dans la plupart des conférences sur l'eau. Il comprend plusieurs principes que nous allons présenter et que nous confronterons à des situations précises d'agriculture irriguée de la rive nord et sud de la Méditerranée. Le premier principe donne la définition du territoire hydrologique, le bassin-versant, dont les contours, en Méditerranée, ne vont jamais de soi. Le deuxième principe considère l'unité de la ressource et sa gestion concertée entre usagers, planificateurs et décideurs, une manière technocratique de voir les sociétés alors que celles-ci sont composites dans un territoire donné de la Méditerranée et qu'elles abordent les eaux au pluriel, selon leurs origines, leurs accès, leurs emplois et réemplois. Le troisième principe centre les questions sociales sur les femmes garantes de la vie, mais cette approche masque en réalité les inégalités extrêmes qui se sont développées dans les sociétés rurales méditerranéennes et débouche sur des avancées très limitées pour la gestion des eaux agricoles par les femmes. Le quatrième principe, celui pour lequel Dublin est la référence, est la fixation d'une valeur économique à l'eau. Pourtant cette ressource peut être partagée selon quatre modalités : le péage, le bien commun, le bien public et le marché. En réalité, ces quatre formes coexistent dans le même espace de gestion des eaux, particulièrement en agriculture méditerranéenne. Chaque espace a construit un compromis institutionnel et économique mais l'effet des politiques mondiales de l'eau entraîne, d'une part une précarisation hydraulique pour les producteurs les plus faibles en zones irriguées, et d'autre part une redistribution où l'État, loin d'être désengagé, exproprie les droits d'eau anciens et attribue des ressources excessives aux véritables surconsommateurs des eaux.
\end{abstract}

Mots clés : conservation de l'eau ; gestion des ressources ; irrigation ; mondialisation ; région méditerranéenne.

Thèmes : systèmes agraires ; eau ; économie et développement rural.

\section{Abstract \\ The contradictions in the integrated management of water resources in irrigated Mediterranean agriculture}

The water resources integrated management model is based on principles set in Dublin in 1992 and reproduced under equivalent terms in most Water Conferences through the last 15 years. This paper introduces several of these principles and discusses them against specific irrigation situations in northern and southern Mediterranean countries. The first principle gives the definition of the hydrologic territory, the watershed, whose limits are never really clear in the Mediterranean context. The second principle considers the unity of the resource and its concerted management between users, planners, and deciders. It is a technocratic approach to observing composite human societies in Mediterranean territories compiling a variety of water concerns according to such concepts as origin, access, use, and reuse. The third principle focuses on the social issues on women, the part of society responsible for assuring the basic foundations of life. Still, in reality this approach hides the strong disparities which have developed in rural Mediterranean societies and which have produced very limited results as far as improved water 
management by women is concerned. The fourth principle, for which Dublin is the main reference, is the fixing of an economic value for water. There are four modes commonly used for sharing this resource: instituting the payment of tolls, classifying the resource as common property, administering the resource as a public good, and treating the resource as a good on the market. In actual fact, these four modes co-exist in the same areas of water management, especially in Mediterranean agriculture. Each area has developed an institutional economic compromise, but world water policies lead to the hydraulic impoverishment of the weakest producers in irrigated lands and to a new system for distributing resources in which the State, rather than disengaging from management of the resource, is expropriating the traditional holders of water rights and transferring them to the very people who already over-consume the resource.

Key words: globalization; irrigation; Mediterranean region; resource management; water conservation.

Subjects: farming systems; water; economy and rural development. es débats sur l'eau ont pris une dimension mondiale depuis 15 ans, à l'occasion des grandes conférences sur l'environnement, puis plus spécifiquement sur la ressource en eau. Des forums, des conférences, des rencontres professionnelles se succèdent autour de thèmes, de crises climatiques, seuils critiques, guerres de l'eau annoncées, dont le principal responsable est l'irrigation trop consommatrice d'eau. Le contexte méditerranéen offre un cadre adéquat pour comprendre comment l'agriculture irriguée se transforme et les effets des principes mondiaux de bonne gouvernance de l'eau (Abu Zeid et Afifi, 2006 ; Fourth World Water Forum, 2006 ; World Bank, 2006).

\section{Un climat contraignant et des espaces ruraux transformés par des siècles d'interventions hydrauliques}

Malgré des différences d'intensité entre les rives nord et sud, les pays méditerranéens connaissent un climat marqué par des périodes de fortes sécheresses (plus de 4 mois sur la rive sud), avec des précipitations irrégulières et souvent violentes avec parfois de fortes inondations. La concentration des pluies sur un petit nombre de jours (50 à 100 par an en moyenne) est la règle générale avec des intensités journalières souvent fortes. L'évaporation réduit l'efficacité des pluies et est partout élevée. Compenser localement les déficits par des apports artificiels d'eau fait partie des cultures méditerranéennes. Différentes civilisations ont transformé les contraintes des milieux naturels en modifiant le cours des eaux, en aménageant l'espace, en jouant sur les complémentarités entre montagnes, plaines et deltas. Les activités agricoles se sont organisées selon une trilogie: la céréaliculture sèche, le pastoralisme et l'agriculture irriguée. Les canaux, les aqueducs et autres ouvrages hydrauliques ponctuent la vie et le paysage méditerranéens.

Cependant, la région méditerranéenne n'est pas homogène. Elle est constituée d'arrangements très divers entre zones sèches et zones humides. On y trouve des marais, des lagunes littorales ou des plaines largement arrosées par des fleuves (la vallée et le Delta du Nil en Égypte ou, la plaine du Gharb, au Maroc submergée régulièrement par l'oued Sebou). On peut trouver des plaines semi-arides où les pluies sont irrégulières entre 250 et $500 \mathrm{~mm}$ par an en moyenne, des régions semi-humides entre 500 et $800 \mathrm{~mm}$ de pluies avec un relief varié de plaines, de piedmonts ou de plateaux puis, au-dessus, des régions montagneuses considérées comme de véritables châteaux d'eau naturels. Ces dispositifs varient d'un pays à l'autre et, dans chaque pays, d'une vallée à l'autre, mais tous les cours d'eau permanents ou temporaires ont connu des transformations tout au long de l'histoire pour sécuriser les productions alimentaires et éviter les famines provoquées par les sécheresses. La vallée de la Têt dans les Pyrénées orientales, en France, est un exemple de ces vallées transformées par des centaines de petits canaux d'irrigation en montagne et de grands canaux arrosant la plaine du Roussillon. Les vallées du Segura ou du Jucar, en Espagne, présentent des dispositifs similaires. Les communautés rurales s'arrangent selon des règles souvent informelles pour gérer les eaux, en particulier en période d'étiage. Les rapports entre communautés autour des eaux sont intenses et donnent lieu à des tensions mais aussi des solidarités et des coopérations. La gestion communautaire s'est partout construite sur les conflits d'usage et sur une subsidiarité active (Ruf, 1998).

\section{Une intégration économique ancienne en Méditerranée}

Au milieu du XIX ${ }^{\mathrm{e}}$ siècle, l'économie des pays méditerranéens a profondément changé. Le développement du commerce entre le Nord et le Sud a contraint l'agriculture irriguée à se spécialiser dans des cultures spéculatives, provoquant de la concentration foncière et des besoins en nouvelles terres irriguées. Les pays se sont alors lancés dans de grands travaux de drainage et d'irrigation. Sur la rive sud, l'ampleur de ces travaux s'accentue au $\mathrm{XX}^{\mathrm{e}}$ siècle, après l'indépendance des pays du Maghreb. Céréaliculture sèche, pastoralisme, agriculture irriguée, zones humides résiduelles n'apparaissent plus 
complémentaires mais concurrentes, d'autant plus que l'urbanisation et le tourisme viennent bouleverser les priorités et les traditions, en privilégiant les grandes plaines et les littoraux par rapport aux arrière-pays. La gestion des ressources naturelles est alors fortement étatisée, aboutissant dans le domaine des grands périmètres irrigués à une gestion administrative et centralisée de l'eau.

À la fin du $\mathrm{xx}^{\mathrm{e}}$ siècle, les questions hydrauliques rentrent dans l'arène de la mondialisation. L'espace méditerranéen se trouve confronté, aux influences politiques et économiques mondiales. Sur l'eau, les débats d'orientation des politiques publiques et des modes de gestion prennent une ampleur exceptionnelle, dans la mesure où les inégalités d'accès à l'eau potable et à l'assainissement placent les gouvernements de tous les pays devant une responsabilité majeure. En Méditerranée, comme dans d'autres régions, l'eau pour l'agriculture est mise en question: on assiste à des modifications des prélèvements sur les ressources superficielles (redistribution des terres et des accès aux eaux) et des transferts compensateurs organisés par l'État entre bassins, comme au Maroc et en Espagne. Le recours aux ressources souterraines non renouvelables se réalise par des extractions excessives liées aux initiatives privées individuelles, favorisées par les évolutions technologiques et les politiques publiques. En définitive, les espaces hydroagricoles méditerranéens se fractionnent et se recomposent en territoires disjoints et inégaux par rapport aux accès aux différentes eaux mobilisées. Face aux menaces de pénurie générale, le message standard renvoie à un modèle de gestion de l'eau fondé sur l'idée de l'unicité de la ressource, dont les principes ont été formulés dans des instances internationales et dont l'application s'avère inadaptée au contexte méditerranéen.

\section{La formulation de principes spécifiques}

\section{pour le secteur de l'eau}

L'année 1992 est l'année de référence du début de la période de restructuration du secteur de l'eau à l'échelle mondiale. Ce n'est pas tant la Conférence de Rio de
Janeiro qui s'est tenue en juin 1992 qui retient l'attention, mais plutôt la Conférence internationale sur l'eau et l'environnement de janvier 1992 à Dublin. Celle-ci met l'accent sur la rareté de l'eau douce, son unicité et formule quatre principes directeurs qui vont influencer l'ensemble des politiques publiques de l'eau.

Depuis deux décennies les pays de la rive sud de la Méditerranée pratiquant de l'irrigation ont été invités par la Banque mondiale et le Fonds monétaire international (FMI), au travers d'ajustements structurels, à diminuer leurs dépenses en se désengageant des organismes publics d'intervention, dans le domaine agricole notamment. La nouvelle orientation visait la redéfinition du rôle des structures de gestion et de développement agricole mises en place au sortir des indépendances. La demande des bailleurs de fonds était une responsabilisation des agriculteurs dans la prise en charge des infrastructures d'irrigation. Un certain nombre de conférences internationales dont la Déclaration de Turin de 1999 ont fait de la gestion de l'eau pour l'irrigation une priorité dans les plans d'action. En 2002, le Forum de Fiuggi sur les avancées de la gestion de la demande en eau souhaite une mise en œuvre de stratégies nationales et des dispositifs institutionnels mais les connaissances sur la demande en eau et son utilisation sont imparfaites et trop globales (Margat et Treyer, 2004).

L'irrigation étant perçue comme responsable des grands déséquilibres hydriques du monde, l'effort doit porter sur l'irriguant et les politiques nationales de l'eau s'engagent partout à en limiter la part, en forçant sur trois domaines clés :

- traduire la rareté de l'eau en termes de gestion de la demande, en vue de limiter les usages et de la faire payer sur un marché concurrentiel ;

- mettre en place de nouvelles institutions à l'échelle des bassins hydrographiques, avec mandat de régulation et d'organisation des allocations ;

- mettre en place des associations d'irriguants pour alléger les charges de contrôle et responsabiliser les populations locales amenées progressivement à " payer l'eau au prix de l'eau ".

Dans la plupart des pays méditerranéens, le premier effort technico-économique du passage d'une gestion de l'eau par l'offre à une gestion par la demande ne s'est pas réellement produit. Loin de limiter les prélèvements et de faire jouer des concurrences, la course à la mobilisation des eaux s'est poursuivie et tous les acteurs y contribuent. Ainsi, en Égypte, l'État investit dans des projets de bonification des terres désertiques au sud-ouest du pays avec l'une des plus grosses stations de pompage du monde délivrant l'eau du barrage d'Assouan à de grandes entreprises privées. Au sud de la Tunisie, des villageois multiplient les forages dans les nappes profondes non renouvelables. La mise en place d'institutions nouvelles coordinatrices de bassins-versants est assez générale, sauf en Égypte où la coordination hydraulique est déjà assurée par un ministère spécialisé créé dès le XIX ${ }^{\mathrm{e}}$. siècle. Les nouvelles agences de bassin éprouvent des difficultés relatives à leur insertion dans des dispositifs étatiques existants, en particulier dans les domaines de compétence contestés entre les administrations et les ministères, à leur financement et leurs réelles possibilités d'action. Ces agences de l'eau créées à partir du modèle français ont du mal à se conformer rapidement à des dispositifs difficiles à mettre en place.

Quant à la mise en place des institutions locales devant assurer une participation active des populations à la gestion de l'eau, elle se trouve freinée par différents facteurs historiques, économiques et sociaux.

\section{Une mosaïque de situations spécifiques et un seul modèle de réforme du secteur de l'eau}

Les situations actuelles correspondent à des combinaisons entre l'irrigation traditionnelle ancienne gérée collectivement et l'irrigation moderne gérée de façon collective ou individuelle. Il existe une gamme de dispositifs gravitaires anciens de mobilisation des eaux de surface, comme, les acequias-fesas en pays valencien, seguias-targas au Maroc, agouilles en Roussillon, raya-mesqa-saqqia en Égypte. On trouve aussi des dispositifs de captation des eaux souterraines appelés mines d'eau sur la rive nord, khetarra au Maroc, foggara en Algérie et des dispositifs d'exhaure, les norias, présentes sur tout le pourtour méditerranéen. D'autres systèmes se sont mis en place avec les 
pompes diesels et électriques relevant l'eau des rivières, des canaux ou des nappes phréatiques, et plus récemment l'eau de nappes non renouvelables. De nombreux réseaux d'irrigation dans la zone méditerranéenne ont été créés avant l'établissement des services étatiques hydrauliques et sont gérés suivant des règles communautaires locales, souvent remaniées et modernisées.

Parallèlement à ces évolutions des réseaux anciens collectifs, les administrations de l'eau engagent depuis 20 ans les agriculteurs des grands périmètres publics à se constituer en associations d'irriguants pour que leur pays puisse bénéficier des prêts des bailleurs de fonds internationaux qui conditionnent leur aide au développement par l'instauration de réformes comme le transfert de gestion ou la gestion participative de l'irrigation.

Or le schéma imposé est partout le même: l'association d'irriguants "type " est constituée sur des bases voisines de celles de la loi française des associations 1901 (Valony, 2004). Élaboré dans des conditions historiques différentes et dont le but unique était de s'organiser pour effectuer des travaux de réfection ou d'agrandissement de réseaux, ce modèle est appliqué à l'ensemble des pays, quel que soit leur système politique, leur mode de gestion des eaux et des terres. Jaubert de Passa, en 1845, écrivait justement dans son étude des civilisations hydrauliques " qu'aucun modèle normatif ne doit s'appliquer à toutes les situations du monde et qu'il faut laisser à l'assemblée des usagers de l'eau le soin d'organiser leurs institutions" (Mollard, 2004). En imposant la mise en place d'associations d'usagers comme une condition formelle d'octroi de prêts pour la poursuite des investissements hydrauliques agricoles, les instances internationales ont incité les administrations des eaux à fabriquer des associations prétextes ou des associations dont l'activité est extrêmement limitée. La délimitation du périmètre d'action de l'association est souvent inadaptée aux contextes sociaux villageois et aux architectures des réseaux de canaux. Ces délimitations artificielles ne s'appuient pas sur les instances de régulation préexistantes. Très souvent institutions anciennes et modernes s'ignorent, quand elles n'entrent pas directement en conflit. Le cadre statutaire est souvent imposé, la langue de l'écrit n'étant celle parlée par les paysans. La possibilité de forger des règlements intérieurs est envisageable mais rarement mise en pratique, dans la mesure où la formation même de l'association obéit à des exigences extérieures à la société locale et même à l'administration du pays. Les associations de gestion de l'eau sur la rive nord de la Méditerranée sont anciennes et on a pris le temps de faire passer les réformes auprès des populations. Ce processus d'intégration institutionnelle s'est déroulé sur plusieurs décennies et, comme pour les agences de bassin, les incitations politiques et économiques sur le court-moyen terme semblent mal adaptées.

La remise en cause des modèles dits traditionnels depuis les années 1960 a conduit à des transferts techniques modernes dont la création de périmètres de grande hydraulique avec des infrastructures importantes et des prélèvements très élevés sur les ressources en cohérence avec les politiques agricoles des pays et leurs besoins. La demande en eau a augmenté de façon considérable. Dans la plupart des pays, les zones irriguées sont composées de nombreuses et petites exploitations en irrigation gravitaire et de grandes exploitations pratiquant sur des superficies étendues l'irrigation en aspersion ou au goutte à goutte. Les crises de surexploitation proviennent de toutes ces dynamiques mais les causalités sont mal élucidées et les solutions en contradiction avec les faits

\section{Des principes mondiaux et des réalités des agricultures irriguées méditerranéennes}

Pour comprendre comment les crises hydrauliques et agricoles se propagent dans presque tous les sites de la Méditerranée, il nous semble important de reprendre l'analyse détaillée du texte qui souligne les contradictions et les enjeux de l'eau au XxI ${ }^{\text {e }}$ siècle (d'après Ruf, 2006).

\section{Premier principe de Dublin}

Ce premier principe affirme que l'eau, ressource fragile et non renouvelable, est indispensable à la vie, au développement et à l'environnement. Il introduit égale- ment les notions de bonne gestion des ressources sous l'angle de la conciliation entre le développement socio-économique et la protection des écosystèmes naturels et de gestion efficace du sol et de l'eau pour la totalité du bassin-versant ou d'un aquifère.

Le caractère non renouvelable semble en contradiction avec le cycle naturel de l'eau. La plus grande partie des eaux douces est constamment renouvelée massivement par le jeu de l'évaporation et de l'évapotranspiration et celui des précipitations et du mouvement mêmes des masses d'eau. La notion d'efficacité relative à une aire hydrologique particulière est avancée sans être définie, d'autant plus que les deux aires de gestion, le bassinversant total et l'aquifère coïncident rarement. C'est l'entité du bassin-versant qui est retenue comme unité de gestion intégrée, ce qui semble paradoxal car les principaux problèmes environnementaux depuis 15 ans touchent aux épuisements des nappes souterraines et/ou à leur contamination élevée. Cet engouement sur l'unité hydrologique de surface est au cœeur de toutes les politiques mondiales de l'eau, avec une contradiction majeure : ne plus gérer l'eau par l'offre mais par la demande. Le bassin-versant est une notion relative au contexte d'intervention et aux intérêts concurrents sur les eaux (Ruf, 2004). Il est loin d'être une unité géomorphologique neutre, c'est une unité hydropolitique (Trottier, 2004) où s'affrontent des groupes aux intérêts divergents qui ne partagent pas les mêmes critères d'efficacité. Sur le terrain, la définition même du bassin-versant est souvent difficile à faire. Dans les montagnes, on trouve une juxtaposition de petits bassins-versants unis simplement par un exutoire de collecte des eaux, mais plusieurs emboittements sont possibles en fonction du choix de ce qui est l'exutoire principal. En plaine, les fleuves constituent des axes importants, mais la complexité provient des affluents et des interfluves. Dans les deltas, la question pertinente n'est plus dans l'idée d'unité spatiale de collecte, mais dans l'organisation de l'éparpillement des eaux dans les branches multiples. En réalité, pour ce qui est de l'emploi des eaux par les hommes, ce qui est le plus significatif est l'organisation du prélèvement et de l'épandage des eaux dans les territoires montagneux, les plaines et les deltas via des canaux. L'idée de déversements organisés s'est transformée en notion de "bassin-déversant " au même titre que le 
bassin-versant (Ferraud, 2006). On voit ici que ces notions doivent être plurielles : il y a des bassins-versants emboités et des "bassins-déversants" successifs et interdépendants (Ruf, 2001). La "bonne gestion des ressources" prend-elle en compte cette complexité spatiale, sociale, hydrologique, hydraulique et politique?

\section{Deuxième principe de Dublin}

Ce principe aborde les questions des décisions politiques. Il annonce en effet que la gestion et la mise en valeur des ressources en eau doivent associer usagers, planificateurs et décideurs à tous les échelons. Il introduit également l'idée de prise de conscience de l'importance des ressources en eau et le principe de subsidiarité, en employant toutefois le conditionnel : les décisions seraient donc prises à l'échelon compétent le plus bas le plus pertinent en accord avec l'opinion publique et en associant les usagers à la planification et à l'exécution des projets relatifs à l'eau.

Qui est usager, planificateur et décideur dans nos sociétés actuelles? À l'échelle villageoise dans le monde rural, ou à l'échelle du quartier d'une ville, doit-on envisager de ne parler que d'usagers? Les hommes et les femmes qui composent la société rurale et la société urbaine sont aussi des citoyens, électeurs éventuels de représentants légitimes chargés d'administrer pour le bien public et commun les ressources locales. Ce sens restreint donné aux utilisateurs locaux de ressources a abouti à mettre en place au Maroc par exemple, dans une démarche très descendante, des associations d'usagers de l'eau agricole, qui s'avèrent être, avec le temps, des instances aux pouvoirs limités, et sans réelle possibilité de se constituer en association de défense des droits des familles rurales (Raki et Ruf, 2004).

La terminologie des débats internationaux sur l'eau tend à vider de sens les enjeux sociaux, les identités locales et régionales, les particularismes historiques. Usager, planificateur, décideur de l'eau, c'est la nouvelle trilogie du langage et de la représentation de la société rurale qu'imposent les politiques publiques. Dans la pratique, associer les usagers à la planification et à l'exécution de projets dans le domaine de l'irrigation se résume le plus souvent à leur demander une contribution financière. Certes, il y a des exemples d'interventions plus complexes avec l'émergence d'associations d'intérêt commun, mais ces exemples sont généralement cantonnés à l'échelon local du petit périmètre irrigué ou du quartier périphérique. Il est rare de voir un développement mutualiste régional de grande envergure dans la gestion des eaux urbaines ou des eaux pour l'agriculture, en particulier dans les pays du Sud.

Le principe de subsidiarité, repris depuis 1992 dans la plupart des conférences internationales, comporte une ambiguité majeure. En principe, il s'agit de laisser une autonomie importante à une communauté inscrite dans un territoire pour gérer ses ressources, dès lors que le niveau plus central est moins bien armé pour le faire. Mais qui est l'entité centrale et qui est l'entité locale? Dans de nombreux cas, l'interprétation technocratique met en scène le ministère de l'Eau d'un côté et les agences ou offices régionaux de l'autre, en oubliant toute autre échelle plus locale, en particulier celle des villages et des associations.

\section{Troisième principe de Dublin}

Le troisième principe s'intéresse aux femmes qui jouent un rôle essentiel dans l'approvisionnement, la gestion et la préservation des eaux. Il fait écho au fait que les arrangements institutionnels tiennent rarement compte du rôle primordial des femmes comme utilisatrices de l'eau et gardiennes du milieu vivant.

Si les questions relatives à l'accès à l'eau pour les femmes sont évidemment de grande importance, l'accent insistant depuis 15 ans dans ce domaine a occulté l'ensemble des questions sociales de la gestion de l'eau. Pour l'agriculture, malgré 20 ans d'affichage gender, rares ont été les projets d'irrigation qui ont véritablement ouvré avec des moyens adéquats pour que les femmes disposent de droits reconnus et de représentations effectives. En revanche, les réseaux villageois d'eau potable ont bien progressé sous l'influence des femmes rurales qui ont pu prendre leur part dans l'organisation et faire valoir leurs droits. Mais en agriculture irriguée, aucune obligation de réforme n'a tenté de faire modifier les statuts locaux (mode d'héritage, nombre d'heures de travail, salariat) des femmes cultivatrices de champs et de jardins, alors que l'on a su imposer des associations d'usagers de l'eau comme condition de la poursuite des financements des projets hydroagricoles.

Les femmes ont ainsi toujours beaucoup de mal à trouver une place entre des instances anciennes qui les ignorent en partie et des instances nouvelles dont la légitimité et la capacité d'action sont faibles. Mais plus globalement, la création de nouvelles institutions s'opère sans tenir compte des inégalités persistantes entre riches et pauvres, propriétaires fonciers et locataires, familles issues de vieilles noblesses et familles sans terre, usuriers et endettés, sédentaires et nomades, etc.

\section{Quatrième principe de Dublin}

L'eau utilisée à de multiples fins, a une valeur économique et devrait donc être reconnue comme bien économique. La valeur économique de l'eau a été longtemps méconnue, ce qui conduit à gaspiller la ressource et à l'exploiter au mépris de l'environnement.

C'est ce dernier principe qui risque de mettre en péril l'économie des familles paysannes qui pratiquent l'irrigation depuis des générations, sur des territoires artificialisés par des décennies de travaux anciens. L'Égypte constitue l'un des pays méditerranéens en déphasage avec cet énoncé dans la mesure où l'eau est distribuée gratuitement aux agriculteurs dans la vallée et le delta du Nil. Mais en réalité, les paysans égyptiens ont contribué plusieurs fois au paiement des dispositifs qui leur apportent l'eau du fleuve. Sous le règne de Mohammed Ali (1805-1849), ils ont été des centaines de milliers mobilisés pour chaque chantier de creusement de grand canal d'irrigation et pour l'édification du premier barrage du delta. Ils ont en outre livré le coton imposé par le souverain aux bénéficiaires du réaménagement des rives du Nil. Après la banqueroute de l'Égypte en 1879 et l'instauration du régime colonial (jusqu'en 1914), les paysans ont payé en argent le remboursement de la dette aux banques européennes, la mise en place de services administratifs hydrauliques modernes et la construction du premier barrage réservoir d'Assouan. Sous le régime de Nasser (1952-1970), ils ont versé toute la production cotonnière aux coopératives d'État pour permettre au gouvernement égyptien indépendant de payer le haut barrage d'Assouan. Enfin, au cours de ces deux siècles de transformation hydraulique et politique, les paysans ont assumé le coût de l'exhaure de l'eau depuis les canaux jusqu'à leur champ grâce à des centaines de milliers de norias mues par les animaux. Depuis 20 ans, ils assument tou- 
jours cette charge avec des pompes diesels mobile. L'eau n'est donc pas vraiment un don du Nil ou un bien gratuit mais s'acquiert par l'investissement collectif et individuel, et par des coûts élevés de transaction et de fonctionnement énergétique (Ferraton et al., 2004 ; Ruf, 1988 ; Ruf, 1996).

Considérer l'eau comme un bien économique et la gérer en conséquence, c'est ouvrir la voie à une utilisation efficace et à une répartition équitable de cette ressource, à sa préservation et à sa protection, insiste le commentaire du quatrième principe dans la déclaration de Dublin. Depuis 15 ans, on a le recul nécessaire pour juger de la pertinence de la déclaration.

Renchérir le prix de l'eau aboutira-t-il à du développement durable? La constitution de réservoirs et de barrages a retiré aux paysanneries une partie significative des eaux qu'elles utilisaient sous des régimes certes plus aléatoires, mais auxquels elles avaient adapté leurs pratiques. Que se passe-t-il aujourd'hui en termes d'efficacité économique? Les eaux stockées s'évaporent en partie. Ce n'est pas la question principale. Elles constituent une masse inerte, objet de nouvelles convoitises. On va décrier les anciens utilisateurs à l'aval immédiat des barrages en stigmatisant leur technique arriérée d'épandage gravitaire ou d'irrigation à la raie, pour tenter de prendre possession d'une partie de la ressource en vue d'extensions nouvelles et modernes. En définitive, on agrandit surtout vers l'aval des périmètres anciens les réseaux d'amenée d'eau en créant un espoir pour des agriculteurs mal desservis les années sèches et en provoquant une forte spéculation foncière et l'arrivée de nouveaux opérateurs investisseurs.

À force de proposer de l'eau aux anciens utilisateurs comme aux nouveaux consommateurs, l'administration hydraulique est prise entre des contradictions fortes: offrir de l'eau régularisée aux anciens utilisateurs du cours d'eau à condition que ceux-ci acceptent que l'eau ait une valeur économique et donc versent une redevance à l'État. Or, bien des paysans résistent effectivement aux conditions proposées et, s'ils sont suffisamment riches, s'équipent de puits et de pompes pour capter l'eau de la nappe phréatique s'affranchissant ainsi de la décision du barragiste. Dans le même temps, l'État, tenté par la surestimation des ressources hydriques sur lequel il exerce le pouvoir de gestion, pousse au développement de nouveaux usages (les golfs par exemple) pour lesquels la valorisation de l'eau ne pose pas de problèmes particuliers. Mais ces opérateurs s'aperçoivent rapidement des défaillances du système de régulation et des risques qu'ils prennent à investir sur la seule base de l'eau publique payante. Ils investissent massivement dans les forages profonds, dans des nappes non renouvelables, même si le coût d'extraction est dix fois supérieur au coût d'acquisition de l'eau du barrage.

Faut-il, donc, fixer une valeur économique unique à l'eau agricole alors que cette ressource est un bien complexe qui peut être partagé selon quatre modalités : le bien commun, le péage, le bien public et le marché (Ruf, 2002). En réalité, ces quatre formes coexistent dans le même espace de gestion des eaux, particulièrement en agriculture, et fonctionnent avec des contributions financières des agriculteurs sous des formes variées, monétaires, en nature ou de travaux.

\section{Conclusion}

Chaque espace, en Méditerranée, a construit un compromis institutionnel et économique, mais l'effet des politiques mondiales de l'eau entraîne d'une part une précarisation hydraulique pour les producteurs les plus faibles en zones irriguées, d'autre part une redistribution où l'État, loin d'être désengagé, exproprie les droits d'eau anciens et attribue des ressources excessives aux véritables surconsommateurs des eaux. L'agriculture irriguée méditerranéenne n'est pas incompatible avec la gestion intégrée des ressources en eau, mais l'application d'un cadre trop technocratique provoque des contradictions sociales, économiques et environnementales. En tout état de cause, la gestion des eaux pour l'agriculture devrait s'appuyer sur un compromis régional et social en reprenant les critiques des quatre principes de Dublin :

- des territoires intégrant les notions d'espaces composites formés de bassinsversants et " déversants ";

- des associations d'irriguants reconnues d'utilité publique et disposant de l'autonomie financière ;

- des représentations de tous les groupes sociaux dans les institutions d'arbitrage ;

- la reconnaissance de toutes les dimensions économiques de la question de l'eau et notamment la diversité des modes de gestion (mutualisation des charges).

\section{Références}

Abu-Zeid, K, Afifi, SM. Integrated water resources management (IWRM). Séminaire régional sur les plans nationaux de l'eau, Rabat 24-25 janvier. 2006.

Ferraud J. Présentation de la Chambre d'agriculture des Pyrénées-Orientales. Séminaire "Carrefour des associations d'irriguants". Projet euroméditerranéen ISIIMM (Institutionnal and social innovations in irrigation Mediterranean management), Fayoum (Égypte) février 2006

Ferraton N, Ayeb H, Lanau S, et al. La gestion locale de l'eau dans le delta du Nil, étude du système d'El Resqa, province de Beheira, Egypte. Rapport de synthèse Cnearc-IRDUniversité du Caire, 2004.

Fourth World Water Forum. MENA report. Mexico, 2006

Margat J, Treyer S. L'eau des Méditerranéens : situation et perspectives. Plan bleu. MAP Technical Reports Series $n^{\circ}$ 158. Athènes: Programme des Nations unies pour l'environnement (Pnue); Plan d'action pour la Méditerranée (Pam), 2004.

www.planbleu.org/publications/mts158.pdf.

Mollard E. Jaubert de Passa, un précurseur. In : Ruf T, Honegger A, eds. Gestion sociale de l'eau, concepts, méthodes de recherche et applications. Territoires en mutation 2004 ; (12) : 17-31.

Raki M., Ruf T. La participation des usagers de l'eau des périmètres irrigués méditerranéens : regards croisés sur deux modèles marocain et égyptien. Coordinations hydrauliques et justices sociales. Quatrième séminaire du PCSI Agropolis, Montpellier, 25 et 26 novembre 2004.

Ruf T. Histoire contemporaine de l'agriculture égyptienne, essai de synthèse. Collection "Etudes et thèses". Paris : Orstom éditions, 1988.

Ruf T. La participation des fellahs à I'agriculture irriguée dans l'Egypte contemporaine. Houille Blanche 1996 ; (8) : 66-9.

Ruf T. L'eau, une ressource rare stratégique dans le Bassin méditerranéen. Synthèse de l'atelier du séminaire international « Agriculture et développement durable en Méditerranée ". Montpellier, 10-12 mars 1997. Sécheresse $1998 ; 9: 62-6$.

Ruf T. L'eau, l'art et la violence. Un millénaire de confrontations et régulations dans le bassin de la vallée de la Têt (Pyrénées-Orientales). Montagnes méditerranéennes. Politiques de l'eau et développement local, de la réflexion à l'action en milieu méditérranéen 2001: 14: 71-8. 
Ruf T. L'irrigation abordée sous l'angle de la gestion communautaire de biens communs, Cadre conceptuel et méthode d'approche des conflits entre parties prenantes. In : Garin P, Le Gal PY, Ruf T, eds. La gestion collective des périmètres irrigués à l'aube du XXle siècle, penjeux, problèmes, démarches. Collection "Colloques". Montpellier: coéditions CiradCemagref-IRD, 2002

Ruf T. Le système irrigué comme territoire. In : Ruf T, Honegger A, eds. Gestion sociale de I'eau, concepts, méthodes de recherche et applications. Territoires en mutation 2004 ; (12) : 51-62
Ruf T. La gestion participative de l'irrigation, compromis social ou précarité hydraulique? Fausses apparences et vraies redistributions des pouvoirs sur les eaux en général et sur les eaux agricoles en particulier. Colloque " Gestion concertée des ressources naturelles et de I'environnement. Du local au global, pour un dialogue entre chercheurs, société civile et décideurs". Thématique $\mathrm{n}^{\circ} 5$ "Gestion de I'eau pour l'agriculture: quelles modalités de partage des responsabilités entre le public, le privé et le "communautaire ". Université de Versailles-Saint-Quentin-en-Yvelines (UVSQ), 26-28 juin 2006.
Trottier J. Institutional and social innovations in irrigation Mediterranean management Communication orale au séminaire du conseil scientifique du Projet ISIIMM, Trévise, 15 octobre. 2004.

Valony M.J. Des communautés d'irriguants aux associations d'usagers de l'eau: un modèle mondial face aux différentes configurations socio-territoriales. Coordinations hydrauliques et justices sociales. Quatrième séminaire du PCSI Agropolis, Montpellier, 25 et 26 novembre 2004.

Worldbank. MENA development report on water. Washington (DC) : World Bank, 2006. 